\title{
Regenerator Placement Algorithms for Connection Establishment in All-Optical Networks
}

\author{
Sang-Wan Kim, Seung-Woo Seo and Seong Cheol Kim \\ School of Electrical Engineering, Seoul National University, Seoul, Korea 151-742
}

\begin{abstract}
In this paper, we deal with the problem of establishing a lightpath in a multihop manner under physical constraints. We provide both minimal-cost and heuristic algorithms for locating signal regeneration nodes(SRNs). For a minimal-cost algorithm, we formulate the problem using dynamic programming(DP) such that blocking of other lightpaths due to the lack of trans mitters/receivers ( $T X s / R X s)$ and wavelengths is minimized throughout the network. The blocking performances of several algorithms are compared with one another in a ring.
\end{abstract}

\section{INTRODUCTION}

In wavelength-division multiplexing(WDM) all-optical networks, most of the virtual topology design algorithms have ignored physical limitations such as amplified spontaneous emission noise, crosstalk due to signal leakage in OXC's, optical-fiber nonlinearities, dispersions, etc.

In reality, signal degradation can result in a non-zero bit error rate(BER), and thus, when a call request arrives, it may be necessary to estimate the BER of a lightpath before actual establishment. Obviously, in the case when the estimated BER is lower than a given BER requirement, the lightpath can be established in a singlehop, otherwise it should be blocked or established in a multihop manner. For the latter case, the lightpath may require signal regeneration at some intermediate nodes, dividing the lightpath into two or more fragments until each fragment satisfies the BER requirement.

Recently, attempts have been made to consider the virtual topology design under physical constraints [4] [5] [7] Datta et al deal with on-line BER calculation for a lightpath to apply to call admission [4]. In [7], three different approaches are compared based on the number of all-optical fragments in a single lightpath: transparency, opacity and translucency. In the translucent case, if a lightpath is unavailable(because of either unavailable resources or unacceptably high BER), then the lightpath is broken into two fragments at a node half-way along the lightpath. In [5], a heuristic algorithm is proposed that sets up a lightpath in a multihop manner when its estimated BER is higher than a required BER.

In this paper, we deal with the problem of establishing a lightpath in a multihop manner under physical constraints and provide both minimal-cost and heuristic algorithms for locating SRNs. We first formulate the problem using dynamic programming(DP) for minimal-cost placement. It is noted that DP has previously been used for optimal placement of erasure nodes [8] and wavelength converters [9].

This paper is organized as follows. In Section 2, we define the problem we would like to deal with. In Section 3 , we first formulate the problem as DP, and then provide three heuristic algorithms in Section 4. Section 5 shows the performance results in a ring. Conclusions are given in Section 6.

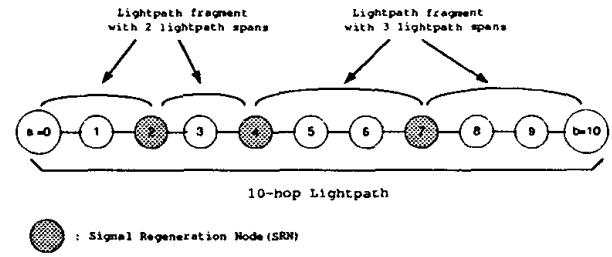

Fig. 1. Illustration of lightpath fragments and lightpath spans in a 10-hop lightpath

\section{Preliminaries}

We first define some notations and terminology used throughout this paper. Let us consider an $H$-hop route between a source node $a$ and a destination node $b$ where nodes along this route are numbered $0,1, \ldots, H$. An endto-end call request along this route is denoted as $\Lambda_{a, b}$. A lightpath fragment is defined as a set of links between two consecutive SRNs. We also define a lightpath span, $L_{s}$, as the maximum number of links along which an optical signal can travel without signal regeneration. The lightpath span indicates the degree of signal degradation, thus varies depending on the characteristics of physical components. Usually, signal regeneration at a node requires a free TX/RX [7] which we assume is tunable. In Fig.1, an illustration of lightpath fragments and lightpath spans in an 10-hop lightpath is shown.

To simplify the formulation, we assume that BER estimation can be obtained on-line through one of the models in previous works [4] [5] [7] using various physical parameters. From these models, we define $B_{i j}$ as a variable indicating whether or not a lightpath between nodes $i$ and $j$ can be established in a single-hop manner, i.e.,

$$
B_{i j}= \begin{cases}1 & \text { if a lightpath satisfies the BER } \\
\infty & \begin{array}{c}
\text { requirement } \\
\text { otherwise. }
\end{array}\end{cases}
$$

Let $h_{i}$ be the $i$ th SRN. For an integer $k$, define a fragment vector $\mathbf{h}=\left(h_{1}, h_{2}, \ldots, h_{k}\right)$ with $0<h_{i}<h_{i+1} \leq H$ where $i=1, \ldots, k-1$. For example, in Fig. $1, \mathbf{h}=\left(h_{1}=\right.$ $2, h_{2}=4, h_{3}=7$ ). We consider $R+1$ SRNs (i.e., $k=R+1$ ) which are placed along the $H$-hop lightpath assuming the destination node $H$ is the $(R+1)$ th SRN. We define $\theta(i, j)$ as a cost which is required to set up a lightpath fragment from node $i$ to node $j$. The goal then becomes finding the fragment vector which will bring a minimal aggregate cost. Mathematically, for $\mathbf{h}=\left(h_{1}, h_{2}, \ldots, h_{R+1}\right)$, the objective function to minimize can be defined as

$$
\min _{1 \leq R \leq H-1} \sum_{i=0}^{R} B_{h_{i} h_{i+1}} \theta\left(h_{i}, h_{i+1}\right) .
$$


In the case where $\Lambda_{a, b}$ must be set up in a multihop manner, the total number of possible multihop establishments for the call request is $2^{H-1}$ since every node can be a candidate node for signal regeneration. The above problem can be solved by exhaustively searching the entire search space. However, since such an approach is not computationally efficient, we instead provide a more efficient minimal-cost algorithm as well as three heuristic algorithms.

\section{Minimal-Cost Placement Algorithm(MCPA)}

In this section, we provide a solution using the minimalcost placement algorithm(MCPA). In order to formulate the problem as the MCPA, we first define a cost function. This is motivated by the fact that signal regeneration requires additional free TXs/RXs at intermediate nodes, so the careful selection of SRNs is important to minimize the overall blocking of multihop connections. If the traffic rates are uniform in all source-destination pairs, the problem simply reduces to select nodes where more free TXs/RXs are available. In the uniform traffic case, the occupation cost $\Theta(i, j)$ can be defined as follows.

Definition 1: The occupation cost, $\Theta(i, j)$, is a value assigned to a lightpath fragment between nodes $i$ and $j$, and is defined:

$$
\Theta(i, j)=\left\{\begin{array}{l}
\infty \quad \text { if } n_{T X}(j)=0 \text { or } n_{R X}(j)=0 \\
\max \left\{\frac{1}{n_{T X}(j)}, \frac{1}{n_{R X}(j)}\right\} \text { otherwise }
\end{array}\right.
$$

where $n_{T X}(j)$ and $n_{R X}(j)$ are the numbers of unoccupied TXs/RXs of node $j$, respectively.

From the definition of the occupation cost, it is obvious that if the value of $\Theta(i, j)$ is relatively high, node $j$ has a relatively small number of TXs/RXs. If the signal regeneration takes place in a node with a high occupation cost, it is likely to increase the possibility that some new call requests are blocked due to the lack of TXs/RXs. Therefore, SRNs should be the intermediate nodes with low occupation costs to minimize blocking of other call requests. With these observations, the objective function can be set as the minimization of the aggregate occupation cost as follows:

$$
\begin{gathered}
\text { Minimize } \sum_{i=0}^{R} B_{h_{i} h_{i+1}} \Theta\left(h_{i}, h_{i+1}\right) \\
\text { for } \mathbf{h}=\left(h_{1}, \ldots, h_{R+1}=H\right) \text { and } R=1, \ldots, H-1 .
\end{gathered}
$$

For a non-uniform traffic case, the occupation cost needs to be defined differently because it is possible that some connection requests whose sources or destinations happen to be the intermediate nodes of a particular lightpath are generated with a higher probability. In such a case, we must take into account not only the number of free TXs/RXs but also the non-uniform traffic rates among the intermediate nodes.

To define the occupation cost for non-uniform traffic, we assume a dynamic circuit-switched traffic model. In this model, connection request arrivals are typically modeled as a Poisson process, and connection holding times are assumed to be exponentially distributed. Let $\lambda_{i}$ be the average rate of Poisson call arrivals at node $i$ for $i=1, \ldots, N$ where $N$ is the number of nodes in the network. We denote by $l_{i j}$ the probability that a call generated at node $i$ is destined to node $j$, where $i, j=1,2, \ldots, N, l_{i i}=0$, and $\sum_{k=1}^{N} l_{i k}=1$ for all $i$. Then, traffic flow from node $i$ to node $j$ is also Poisson with a rate $t_{i j}=\lambda_{i} l_{i j}$. The set $\Delta_{x i}=\left\{t_{x 1}, t_{x 2}, \ldots, t_{x N}\right\}$ represents $a$ set of traffic rates whose source is node $x$. Similarly, the set $\Delta_{i x}=\left\{t_{1 x}, t_{2 x}, \ldots, t_{N_{x}}\right\}$ represents a set of traffic rates whose destination is node $x$. Using these definitions, we introduce two sets, denoted by $L_{T}(x, k)$ and $L_{R}(x, k)$ :

$$
\begin{aligned}
& L_{T}(x, k)=\left\{p_{T}(x, 1), p_{T}(x, 2), \ldots, p_{T}(x, k) \mid p_{T}(x, m)(5)\right. \\
&\text { is the } \left.m \text { th largest value in } \Delta_{x i}\right\} \\
& L_{R}(x, k)=\left\{p_{R}(x, 1), p_{R}(x, 2), \ldots, p_{R}(x, k) \mid p_{R}(x, n)(6)\right. \\
&\text { is the } \left.n \text {th largest value in } \Delta_{i x}\right\}
\end{aligned}
$$

where $1 \leq m, n \leq k$ and $k=1,2, \ldots, N$. It is noted that $L_{T}(x, k)$ and $L_{R}(x, k)$ have only $k$ largest values in $\Delta_{x i}$ and $\Delta_{i x}$, respectively. Thus, in order to consider a traffic distribution pattern among nodes as well as the number of free TXs/RXs, we sum up the elements of $L_{T}\left(j, n_{T X}(j)\right)$ and $L_{R}\left(j, n_{R X}(j)\right)$ for the numerators of an occupation cost. By taking this ratio, we can give a node with many call requests a relatively high occupation cost. The occupation cost for non-uniform traffic is defined as follows:

Definition 2: The occupation cost, $\Theta^{\prime}(i, j)$, for nonuniform traffic is defined as

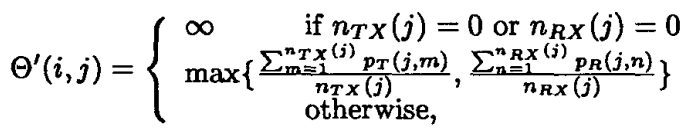

where $p_{T}(j, m) \in L_{T}\left(j, n_{T X}(j)\right)$ and $p_{R}(j, n) \in$ $L_{R}\left(j, n_{R X}(j)\right)$. $n_{T X}(j)$ and $n_{R X}(j)$ are the numbers of unoccupied TXs/RXs of node $j$, respectively.

Obviously, if $\Theta^{\prime}(i, j)$ is substituted for $\Theta(i, j)$ in Equation (4), the objective function minimizes the aggregate occupation cost by considering both non-uniform traffic and TX/RX shortage. Using the occupation cost defined in this section, we present the formulation of the MCPA.

\section{A. Problem Formulation using Dynamic Programming}

Before we proceed, we first provide some definitions.

Definition 3: A set of all fragment vectors with the $m$ th SRN located at node $j$ is defined as

$$
\begin{aligned}
\Psi(m, j) & \equiv\left\{\mathbf{h} \in \mathbf{Z}_{+}^{m}: 0<h_{i}<h_{m}=j, i=1,\right.
\end{aligned}
$$

Definition 4: Chain $[0, j]$ is defined as a set of physical links between nodes 0 and $j$ on $\Lambda_{a, b}$.

Next, in order to formulate the problem into a DP model, three basic elements are defined following the standard notations [11]:

1. For $\mathbf{h} \in \Psi(m, j)$, the stage $m$ is represented by the number of SRNs on a chain $[0, j]$ where $m=$ $1,2, \ldots, H$, and $j=m, \ldots, H$.

2. The alternatives at stage $m$ are represented by the $(m-1)$ th SRNs on a chain $[0, j]$. Thus, there exist $(j-m-1)$ alternatives at stage $m$.

3. The state at stage $m$ is represented by the aggregate occupation cost assigned to stages $1,2, \ldots$ and $m$, combined. During the computation of $\mathrm{DP}$, states should be selected stage by stage so that the aggregate occupation cost is minimized.

Now, we define the minimal aggregate occupation cost, $\alpha(m, j)$. 
Definition 5: For $\mathbf{h} \in \Psi(m, j), \alpha(m, j)$ is defined as the minimal aggregate occupation cost at stage $m$ :

$$
\alpha(m, j)=\min _{m-1 \leq i \leq j-1}\left\{\alpha(m-1, i)+B_{i j} \Theta(i, j)\right\},
$$

for $1 \leq m \leq H$ and $m \leq j \leq H . \alpha(1, j)=B_{0 j} \Theta(0, j)$ for $1 \leq j \leq H$. For non-uniform traffic, $\Theta^{\prime}(i, j)$ should be used instead of $\Theta(i, j)$.

As a result, the minimal aggregate occupation cost is obtained as

$$
\min _{1 \leq i \leq H} \alpha(i, H)
$$

The DP is realized in a bottom-up manner and the running time of the algorithm is $O\left(H^{3}\right)$.

\section{B. Minimal-Cost Placement under Wavelength Con- straints}

When a lightpath is to be set up in a single-hop manner, we usually assume that the same kind of free wavelength is used along the entire source-destination route in case no wavelength conversion mechanism is employed at intermediate nodes. This condition is often called the wavelength-continuity constraint. However, in the case where a connection is established in a multihop manner, each lightpath fragment can be allowed to use different kinds of wavelengths. Therefore, we need to guarantee that the wavelength-continuity constraint is satisfied only within each fragment independently. In other words, even if we obtain the SRNs with the minimal aggregate occupation cost, the lightpath may be blocked due to the lack of wavelengths satisfying the wavelength-continuity constraint within each lightpath fragment. Therefore, in a general situation, the availability of wavelengths should also be considered besides that of free TXs/RXs.

In this case, the occupation cost must be modified to include a factor on the number of free wavelengths on each lightpath fragment. Let $c_{i j}$ be the number of wavelengths which satisfy the wavelength-continuity constraint on a lightpath fragment from node $i$ to node $j$. Also, let $\Delta_{c_{i j}}$ be the set of traffic rates $t_{p q}^{i j}$ for $1 \leq p, q \leq N$ where $t_{p q}^{i j}$ denotes the traffic rate from node $p$ to node $q$ using at least one link on the lightpath fragment between nodes $i$ and $j$. Then, the set $L_{W}$ is defined as follows:

$$
\begin{gathered}
L_{W}(i, j, k)=\left\{p_{W}(i, j, 1), p_{W}(i, j, 2), \ldots, p_{W}(i, j, k) \mid(11)\right. \\
\left.p_{W}(i, j, n) \text { is the } n \text {th largest value in } \Delta_{c_{i j}}\right\}
\end{gathered}
$$

where $1 \leq n \leq k$. We can then define the modified occupation costs, $\bar{\Theta}_{W}(i, j)$ and $\Theta_{W}^{\prime}(i, j)$, as follows.

Definition 6: The occupation costs, $\Theta_{W}(i, j)$ and $\Theta_{W}^{\prime}(i, j)$, are the values assigned to a lightpath fragment between nodes $i$ and $j$, and are defined as

Uniform traffic:

$\Theta_{W}(i, j)=\left\{\begin{array}{c}\infty \quad \text { if } n_{T X}(j)=0 \text { or } n_{R X}(j)=0 \\ \quad \text { or } c_{i j}=0 \\ \max \left\{\frac{1}{n_{T X}(j)}, \frac{1}{n_{R X}(j)}, \frac{1}{c_{i j}}\right\} \text { otherwise }\end{array}\right.$
Non-uniform traffic:

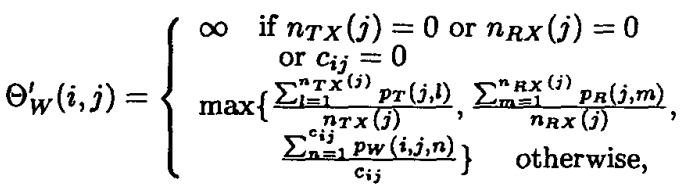

where $p_{T}(j, l) \in L_{T}\left(j, n_{T X}(j)\right), p_{R}(j, m) \in L_{R}\left(j, n_{R X}(j)\right)$ and $p_{W}(i, j, n) \in L_{W}\left(i, j, c_{i j}\right) . n_{T X}(j)$ and $n_{R X}(j)$ are the numbers of unoccupied TXs/RXs of node $j$, respectively.

\section{Heuristic Placement Algorithms}

We next present three heuristic algorithms to place SRNs and derive equations to obtain how many SRNs are required in each algorithm. However, it is noted that these heuristic algorithms may obtain inefficient SRNs because they do not consider the cost of intermediate nodes.

\section{A. Random Placement Algorithm(RPA)}

The random placement algorithm(RPA) chooses the SRNs at random among the intermediate nodes on a lightpath as in Fig.1. The algorithm simply keeps searching recursively until all of the lightpath fragments satisfy the given $B E R$ requirement.

The number of SRNs under the RPA can be obtained using a recurrence relation. We first assume that the lightpath $\operatorname{span} L_{s}$ is fixed on $\Lambda_{a, b}$. Then, we define $N_{r}(a, b)=N_{r}(b-a)$ as the average number of SRNs to establish a lightpath request. When an intermediate SRN $i$ is obtained randomly, $\Lambda_{a, b}$ is divided into two lightpath fragments: one from node $a$ to node $i$ and the other from node $i$ to node $b$. Then,

$$
N_{r}(a, b)=\frac{1}{b-a-1} \sum_{i=a+1}^{b-1}\left[N_{r}(a, i)+N_{r}(i, b)+1\right] .
$$

The initial values of the above equation depend on the value of the lightpath span $L_{s}$. Hence, $N_{r}(1), N_{r}(2), \ldots, N_{r}\left(L_{s}\right)=0$, and $N_{r}\left(L_{s}+1\right)=1$. For example, if $L_{s}=3, N_{r}(1)=0, N_{r}(2)=0, N_{r}(3)=0$ and $N_{r}(4)=1$.

\section{B. Half Placement Algorithm(HPA)}

The half placement algorithm(HPA) chooses the SRN at the midpoint of the lightpath, and keeps searching recursively on each lightpath fragment until all of the lightpath fragments satisfy the given $B E R$ requirement.

As in the RPA, the number of SRNs can also be obtained using a recurrence relation. Defining $N_{h}(a, b)=$ $N_{h}(b-a)$ to be the number of SRNs on $\Lambda_{a, b}$,

$$
N_{h}(a, b)=\left\{\begin{array}{l}
2 N_{h}\left(\frac{b-a}{2}\right)+1 \\
\text { if } b-a \text { is an even number } \\
N_{h}\left(\frac{b-a-1}{2}\right)+N_{h}\left(\frac{b-a+1}{2}\right)+1 \\
\text { if } b-a \text { is an odd number }
\end{array}\right.
$$

For a fixed lightpath span $L_{s}, N_{h}\left(L_{s}\right)=0, N_{h}\left(L_{s}+1\right)=1$, $\ldots, N_{h}\left(2 L_{s}-1\right)=1$ and $N_{h}\left(2 L_{s}\right)=1$. 

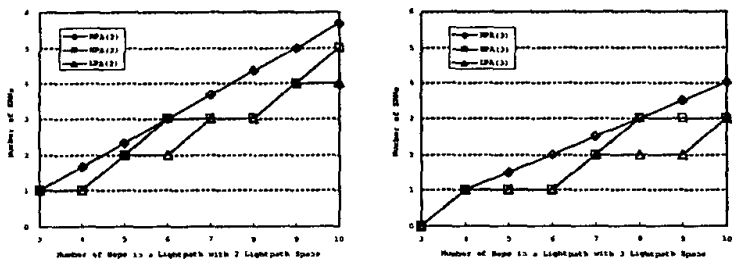

Fig. 2. Number of SRNs in RPA, HPA and LPA for $L_{s}=2$ and 3

\section{Linear Placement Algorithm(LPA)}

The linear placement algorithm(LPA) finds the SRNs in order from source to destination.

The number of SRNs can also be obtained by defining $N_{l}(a, b)=N_{l}(b-a)$ as before for a fixed lightpath span $L_{s}$ as follows:

$$
N_{l}(a, b)=N_{l}(b-a)=\left\lceil\frac{b-a}{L_{s}}\right\rceil-1 .
$$

The performance of the above three heuristic algorithms are compared by the number of SRNs necessary to establish a lightpath under the same fixed lightpath span. In Fig.2, we plot the number of SRNs as a function of the number of hops in a lightpath for $L_{s}=2$ and 3 . The results show that the LPA requires the smallest number of SRNs followed by the HPA and the RPA. It is evident that the shorter the lightpath spans are, the more SRNs are required.

\section{Numerical EXAMPLE}

In this section, we compare the performance of the algorithms presented in this paper. Performance comparison is made by simulation in a 25-node bidirectional ring. The MCPA is compared with the following cases: RPA, HPA, LPA, a full placement(FP) as in fully opaque networks [7] and a case with an ideal physical layer. In FP, all of the lightpath fragments have a single lightpath span, that is, $L_{s}=1$. In the ideal network, the physical constraints are ignored and the network is assumed to set up a connection without any SRNs at intermediate nodes. Throughout the simulations, we assume the lightpath spans are fixed to some values.

The ring is an optical topology with strong correlations among the link loads. Such correlations are helpful in demonstrating a feature of the MCPA, i.e, minimizing the blocking of some other connection requests. In this simulation, we assume that call requests arrive at each node according to a Poisson process. When a call request is generated at a node, it may be destined to the other nodes with equal or different probabilities depending on uniform or non-uniform traffic pattern. $10^{6}$ call requests are generated and the call holding time is assumed to be exponentially distributed. We use the shortest-path fixed routing algorithm to route lightpaths and the first-fit wavelength assignment algorithm in which the lowest indexed wavelength is chosen and assigned among a set of available wavelengths [3].

In Fig.3, we examine the performance of the MCPA by comparing with other algorithms for $4 \mathrm{TXs} / \mathrm{RXs}, 8$ wavelengths and $L_{s}=4$ under uniform traffic. The MCPA

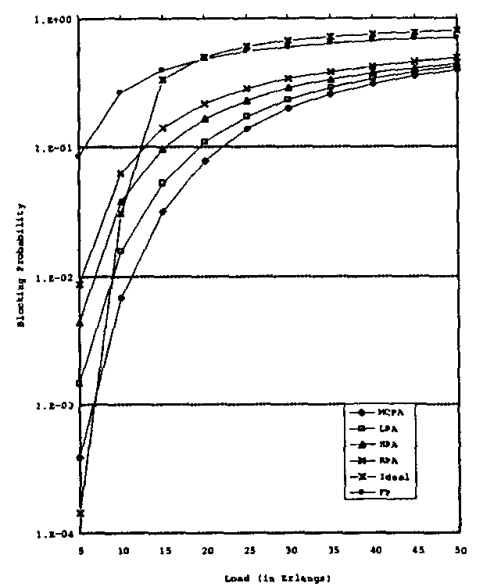

Fig. 3. Blocking probabilities in a 25 -node ring for $\mathrm{TX} / \mathrm{RX}=4$, wavelength $=8$, and $L_{s}=4$

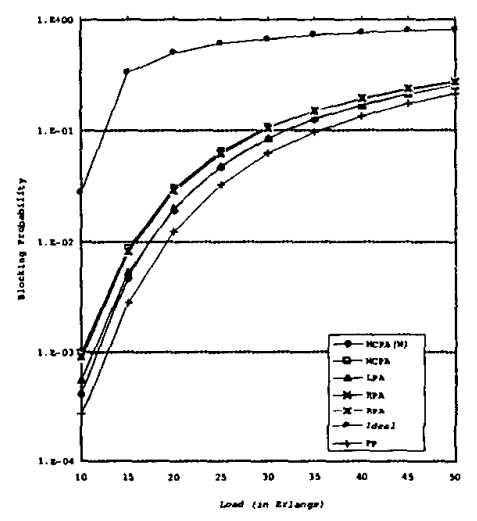

Fig. 4. Blocking probabilities in a 25-node ring for $\mathrm{TX} / \mathrm{RX}=16$, wavelength $=8$, and $L_{s}=4$

shows the best performance as expected, followed by the LPA, HPA, RPA and FP. When the number of TXs/RXs is relatively smaller than the number of wavelengths, the performance of the algorithms depends on the efficient utilization of TXs/RXs. Thus, the MCPA outperforms the other placement algorithms by a significant margin. However, as the number of the TXs/RXs increases, the performance gap among the algorithms decreases because the call blocking is mainly due to the lack of wavelengths, as shown in Fig.4. It is interesting to see that the performance of FP is relatively improved in Fig.4, compared to Fig.3. This is due to the fact that FP uses wavelengths most efficiently with enough TXs/RXs. In Fig.4, we apply the occupation cost $\Theta_{W}(i, j)$ to the MCPA which considers the number of wavelengths in the lightpath fragments. In the figure, it can be seen that the MCPA(W) using $\Theta_{W}(i, j)$ shows better performance than the MCPA using 


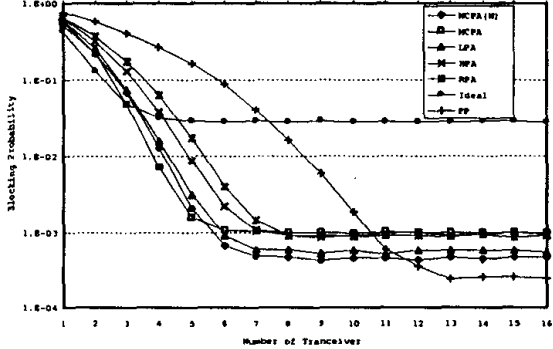

Fig. 5. Blocking probabilities vs. the number of TXs/RXs in 25-node ring for wavelength $=8$, and $L_{s}=4$

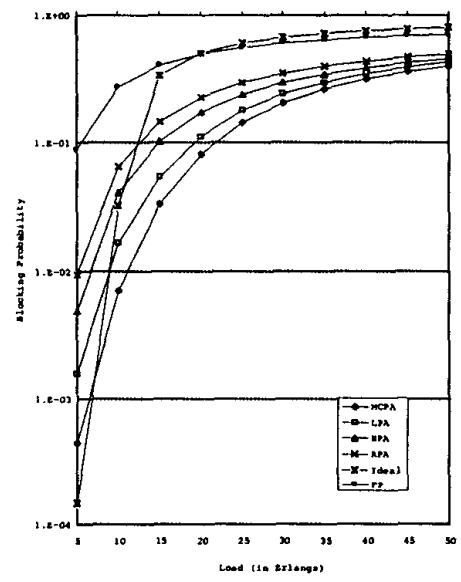

Fig. 6. Blocking probabilities in a 25-node ring for $\mathrm{TX} / \mathrm{RX}=4$, wavelength $=8, L_{s}=4$ under non-uniform traffic

$\Theta(i, j)$.

Fig.5 shows the blocking probabilities as a function of the number of TXs/RXs when traffic loads are $10 \mathrm{Er}-$ langs. For a relatively small number of TXs/RXs, the MCPA using $\Theta(i, j)$ shows good blocking performance. While the performance gap among the MCPA, HPA and RPA decreases as the number of TXs/RXs increases, the MCPA(W) which considers the number of free wavelengths shows good results regardless of the number of TXs/RXs. It is observed that the blocking performance of the ideal case is not so good due to the wavelengthcontinuity constraint as the number of $\mathrm{TX}_{\mathrm{s}} / \mathrm{RX}_{\mathrm{s}}$ increases. On the other hand, the relative throughput gain of FP increases rapidly because a short lightpath fragment reduces the possibility of blocking due to wavelength continuity. For more than $12 \mathrm{TXs} / \mathrm{RXs}, \mathrm{FP}$ shows the lowest blocking probability although this case requires a higher network implementation cost.

For non-uniform traffic, the probability $l_{i j}$ between nodes $i$ and $j$ is generated randomly by satisfying $\sum_{k=1}^{N=25} l_{i k}=1$ for $i=1, \ldots, 25$, while $\lambda_{i}$ 's are assumed to be the same for all nodes. It is again observed in Fig. 6
TABLE I

Running Times of Algorithms

\begin{tabular}{|c|c|c|}
\hline HPA & LPA & MCPA \\
\hline$O\left(\log _{2} \bar{H}\right)$ & $O(H)$ & $O\left(H^{3}\right)$ \\
\hline
\end{tabular}

that the MCPA using $\Theta^{\prime}(i, j)$ shows better performance.

\section{CONCLUSIONS}

In this paper, we study the problem of setting up a lightpath in a multihop manner due to physical constraints. We provide the MCPA which is formulated using DP and three heuristic placement algorithms. For both uniform and non-uniform traffic conditions, the MCPA minimizes blocking of the establishment of other lightpaths due to the lack of free TXs/RXs and wavelengths. Table 1 compares the running times of heuristic algorithms and MCPA. Through simulations, we demonstrate that each algorithm shows various blocking performance according to lightpath spans, the number of TXs/RXs and wavelengths. We show that the MCPA has a better blocking performance than any other heuristic algorithm. In particular, the MCPA is shown to outperform other heuristic algorithms when call requests have a long average hop distance, an appropriate lightpath span and a strong traffic correlation among the link loads.

\section{REFERENCES}

[1] R. Ramaswami and K. N. Sivarajan, "Disign of logical topologies for wavelength-routed optical networks," IEEE Journal on Selected Areas in Communications, Vol.14, No.5, pp.840-851, Jun. 1996.

[2] Z. Zhang and A. S. Acampora "A heuristic wavelength assignment algorithm for multihop WDM networks with wavelength routing and wavelength re-use," IEEE/ACM Trans. on Networking, Vol.3, No.5, pp.281-288, Jun. 1995.

[3] A. Mokhtar and M. Azizoḡlu, "Adaptive wavelength routing in all-optical networks," IEEE/ACM Trans. on Networking, Vol.6, No.2, pp.197-206, April. 1998.

[4] D. Datta, B. Ramamurthy, H. Feng, J. P. Heritage, and B. Mukherjee, "BER-based call admission in wavelength route optical networks," in Proc. OFC'g8, 1998.

[5] J. Y. Youe and S. W. Seo, "An algorithm for virtual topology design in WDM optical networks under physical constraints" Proc.ICC'99, Jun. 1999.

[6] B. Mukberjee, Optical communication networks, New York: McGraw-Hill, 1997.

[7] B. Ramamurthy, H. Feng, D. Datta, J. P. Heritage, and B. Mukberjee, "Transparent vs. opaque vs. translucent wavelengthrouted optical networks", in Optical Fiber Communication (OFC'9g) Technical Digest, San Francisco, Feb. 1999.

[8] B. Narahari, S. Shende, and R. Simha, "Efficient algorithms for erasure node placement on slotted dual bus networks, " IEEE/ACM Than. Networking, Vol.4, No.5, pp.779-784, Oct. 1996

[9] S. Subramaniam, M. Azizog̃lu, and A. K. Somani, "On optimal converter placement in wavelength-routed networks," IEEE/ACM Trans. on Networking, Vol.7, No.5, pp.754-766, Oct. 1999.

[10] Thomas H. Cormen, Charles E. Leiserson, Ronald L. Rivest, Introduction to algorithms, Massachusetts: The MIT Press, 1990.

[11] Hamdy A. Taha, Operations research, New Jersey: Prentice Hall, 1997. 